\title{
UNIFORMLY SUMMING SETS OF OPERATORS ON SPACES OF CONTINUOUS FUNCTIONS
}

\section{J. M. DELGADO and CÁNDIDO PIÑEIRO}

Received 30 March 2004

\begin{abstract}
Let $X$ and $Y$ be Banach spaces. A set $M$ of 1-summing operators from $X$ into $Y$ is said to be uniformly summing if the following holds: given a weakly 1-summing sequence $\left(x_{n}\right)$ in $X$, the series $\sum_{n}\left\|T x_{n}\right\|$ is uniformly convergent in $T \in \mathcal{M}$. We study some general properties and obtain a characterization of these sets when $\mu$ is a set of operators defined on spaces of continuous functions.
\end{abstract}

2000 Mathematics Subject Classification: 47B38, 47B10.

1. Introduction. Throughout this paper, $X$ and $Y$ will be Banach spaces. If $X$ is a Banach space, $B_{X}=\{x \in X:\|x\| \leq 1\}$ will denote its closed unit ball and $X^{*}$ will be the topological dual of $X$. Given a real number $p \in[1, \infty)$, a (linear) operator $T: X \rightarrow Y$ is said to be $p$-summing if there exists a constant $C>0$ such that

$$
\left(\sum_{i=1}^{n}\left\|T x_{i}\right\|^{p}\right)^{1 / p} \leq C \cdot \sup \left\{\left(\sum_{i=1}^{n}\left|\left\langle x^{*}, x_{i}\right\rangle\right|^{p}\right)^{1 / p}: x^{*} \in B_{X^{*}}\right\}
$$

for every finite set $\left\{x_{1}, \ldots, x_{n}\right\} \subset X$. The least $C$ for which the above inequality always holds is denoted by $\pi_{p}(T)$ (the $p$-summing norm of $T$ ). The linear space of all $p$-summing operators from $X$ into $Y$ is denoted by $\Pi_{p}(X, Y)$ which is a Banach space endowed with the $p$-summing norm.

As usual, $\ell_{w}^{p}(X)$ will be the Banach space of weakly $p$-summable sequences in $X$, that is, the sequences $\left(x_{n}\right) \subset X$ satisfying $\sum_{n}\left|\left\langle x^{*}, x_{n}\right\rangle\right|^{p}<\infty$ for all $x^{*} \in X^{*}$; the norm in $\ell_{w}^{p}(X)$ is $\epsilon_{p}\left(x_{n}\right)=\sup \left\{\left(\sum_{n}\left|\left\langle x^{*}, x_{n}\right\rangle\right|^{p}\right)^{1 / p}: x^{*} \in B_{X^{*}}\right\}$. The set of all strongly $p$-summable sequences in $X$ is denoted by $\ell_{a}^{p}(X)$; the norm in this space is $\pi_{p}\left(x_{n}\right)=$ $\left(\sum_{n}\left\|x_{n}\right\|^{p}\right)^{1 / p}$. If $T \in \Pi_{p}(X, Y)$, the correspondence $\hat{T}:\left(x_{n}\right) \mapsto\left(T x_{n}\right)$ always induces a bounded operator from $\ell_{w}^{p}(X)$ into $\ell_{a}^{p}(Y)$ with $\|\hat{T}\|=\pi_{p}(T)$ [5, Proposition 2.1].

Families of operators arise in different applications: equations containing a parameter, homotopies of operators, and so forth. In these applications, it may be very interesting to know that, given a set $\mathcal{M} \subset \Pi_{p}(X, Y)$ and $\left(x_{n}\right) \in \ell_{w}^{p}(X)$, the series $\sum_{n}\left\|T x_{n}\right\|^{p}$ is uniformly convergent in $T \in \mathcal{M}$. The main purpose of this paper is to study uniformly $p$-summing sets, that is, those sets $\mathcal{M} \subset \Pi_{p}(X, Y)$ for which, given $\left(x_{n}\right) \in \ell_{w}^{p}(X)$, the series $\sum_{n}\left\|T x_{n}\right\|^{p}$ is uniformly convergent in $T \in \mathcal{M}$. These sets also enjoy some properties that justify their study; the next proposition lists some of them. 
Proposition 1.1. (a) Let $\left(T_{k}\right)$ be a sequence in $\Pi_{p}(X, Y)$. Then, $\widehat{T}_{k} \stackrel{k}{\rightarrow} 0$ pointwise if and only if $T_{k} \stackrel{k}{\rightarrow} 0$ pointwise and $\left(T_{k}\right)$ is uniformly $p$-summing.

(b) Let $\mathcal{M} \subset \Pi_{p}(X, Y)$ be a uniformly $p$-summing set. If $\mathcal{M}$ is endowed with the strong operator topology, then the map $T \in \mathcal{M} \mapsto \sum_{n}\left\|T x_{n}\right\|^{p} \in \mathbb{R}$ is continuous for every $\left(x_{n}\right) \in$ $\ell_{w}^{p}(X)$.

A basic argument shows that uniformly $p$-summing sets are bounded for the $p$ summing norm. In fact, if $X$ does not contain any copy of $c_{0}$, bounded sets and uniformly 1-summing sets are the same. That is the reason for which we only consider operators defined on a $\mathscr{C}(\Omega)$-space, $\Omega$ being a compact Hausdorff space. We recall that every weakly compact operator $T: \mathscr{C}(\Omega) \rightarrow Y$ has a representing measure $m_{T}: \Sigma \rightarrow$ $Y$ defined by $m_{T}(B)=T^{* *}\left(\chi_{B}\right)$ for all $B \in \Sigma$, where $\Sigma$ denotes the Borel $\sigma$-field of subsets of $\Omega$ and $\chi_{B}$ denotes the characteristic function of $B$. The vector measure $m_{T}$ is regular and countably additive [6, Theorem VI.2.5 and Corollary VI.2.14]. If we denote by $\tilde{T}$ the operator $T^{* *}$ restricted to $B(\Sigma)$ (the space of all bounded Borel-measurable scalar-valued functions defined on $\Omega$ ), then

$$
\widetilde{T} \varphi=\int_{\Omega} \varphi d m_{T}
$$

for all $\varphi \in B(\Sigma)$ (the integral is the elementary Bartle integral [6, Definition I.1.12]).

It is well known that every $p$-summing operator defined on a Banach space $X$ is weakly compact. In Section 2, we consider 1-summing operators $T$ defined on $\mathscr{C}(\Omega)$; these operators are characterized as those with representing measure $m_{T}$ having finite variation and $\pi_{1}(T)=\left|m_{T}\right|(\Omega)$ [6, Theorem VI.3.3]. We show that a set $M \subset \Pi_{1}(\mathscr{C}(\Omega), Y)$ is uniformly 1-summing if and only if the family of all variation measures $\left\{\left|m_{T}\right|: T \in M\right\}$ is uniformly bounded and there is a countably additive measure $\mu: \Sigma \rightarrow[0, \infty)$ such that $\left\{\left|m_{T}\right|: T \in \mathcal{M}\right\}$ is uniformly $\mu$-continuous.

In Section 3, we mention a special class of uniformly $p$-summing operators: uniformly dominated sets. The relationship between uniformly summing sets and relatively weak compactness is also studied. Finally, we give some examples and open problems.

2. Uniformly 1-summing sets in $\Pi_{1}(\mathscr{C}(\Omega), Y)$. Before facing our main theorem, we include three results which correspond to the vector measure theory. These results will be usually invoked along the following lines.

Proposition 2.1 [6, Proposition I.1.17]. The following statements about a collection $\left\{m_{i}: i \in I\right\}$ of $Y$-valued measures defined on a $\sigma$-field $\Sigma$ are equivalent:

(a) the set $\left\{m_{i}: i \in I\right\}$ is uniformly countably additive, that is, if $\left(E_{n}\right)$ is a sequence of pairwise disjoint members of $\Sigma$, then $\lim _{n}\left\|\sum_{k \geq n} m_{i}\left(E_{k}\right)\right\|=0$ uniformly in $i \in I$,

(b) the set $\left\{y^{*} \circ m_{i}: i \in I, y^{*} \in B_{Y^{*}}\right\}$ is uniformly countably additive,

(c) if $\left(E_{n}\right)$ is a sequence of pairwise disjoint members of $\Sigma$, then $\lim _{n}\left\|m_{i}\left(E_{n}\right)\right\|=0$ uniformly in $i \in I$,

(d) if $\left(E_{n}\right)$ is a sequence of pairwise disjoint members of $\Sigma$, then $\lim _{n}\left\|m_{i}\right\|\left(E_{n}\right)=0$ uniformly in $i \in I$, where $\left\|m_{i}\right\|$ denotes the semivariation of $m_{i}$,

(e) the set $\left\{\left|y^{*} \circ m_{i}\right|: i \in I, y^{*} \in B_{Y^{*}}\right\}$ is uniformly countably additive. 
TheOREM 2.2 [6, Theorem I.2.4]. Let $\left\{m_{i}: \Sigma \rightarrow Y: i \in I\right\}$ be a uniformly bounded (with respect to the semivariation) family of countably additive vector measures on $a$ $\sigma$-field $\Sigma$. The family $\left\{m_{i}: i \in I\right\}$ is uniformly countably additive if and only if there exists a positive real-valued countably additive measure $\mu$ on $\Sigma$ such that $\left\{m_{i}: i \in I\right\}$ is uniformly $\mu$-continuous, that is,

$$
\lim _{\mu(E) \rightarrow 0}\left\|m_{i}(E)\right\|=0
$$

uniformly in $i \in I$.

If $\Omega$ is a compact Hausdorff space and $\Sigma$ denotes the $\sigma$-field of the Borel subsets of $\Omega$, a vector measure $m$ on $\Sigma$ is regular if for each Borel set $E$ and $\varepsilon>0$ there exists a compact set $K$ and an open set $O$ such that $K \subset E \subset O$ and $\|m\|(O \backslash K)<\varepsilon$.

Proposition 2.3 [6, Lemma VI.2.13]. Let $\%$ be a family of regular (countably additive) scalar measures defined on $\Sigma$. Each of the following statements implies all the others:

(a) for each pairwise disjoint sequence $\left(O_{n}\right)$ of open subsets of $\Omega, \lim _{n} \mu\left(O_{n}\right)=0$ uniformly in $\mu \in \mathscr{K}$,

(b) for each pairwise disjoint sequence $\left(O_{n}\right)$ of open subsets of $\Omega, \lim _{n}|\mu|\left(O_{n}\right)=0$ uniformly in $\mu \in \mathscr{K}$,

(c) $\mathscr{K}$ is uniformly countably additive,

(d) $\mathscr{K}$ is uniformly regular, that is, if $E \in \Sigma$ and $\varepsilon>0$, then there exists a compact set $K$ and an open set $O$ such that $K \subset E \subset O$ and $\sup _{\mu \in \mathscr{K}}|\mu|(O \backslash K)<\varepsilon$.

Now, we are able to show our main result. In the proof, we will use the fact that $\left|m_{T}\right|$ is regular when $T: \mathscr{C}(\Omega) \rightarrow Y$ is 1-summing [7, Proposition 15.21].

THEOREM 2.4. Let $\mathcal{M} \subset \Pi_{1}(\mathscr{C}(\Omega), Y)$ be a bounded set. The following statements are equivalent:

(a) $M$ is uniformly 1-summing,

(b) the family of nonnegative measures $\left\{\left|m_{T}\right|: T \in M\right\}$ is uniformly countably additive,

(c) given $\varepsilon>0$ and a disjoint sequence $\left(E_{n}\right)$ of Borel subsets of $\Omega$, there exists $n_{0} \in \mathbb{N}$ such that

$$
\sum_{n \geq n_{0}}\left\|m_{T}\left(E_{n}\right)\right\|<\varepsilon
$$

for all $T \in \mathcal{M}$.

Proof. (a) $\Rightarrow(\mathrm{b})$. According to [6, Lemma VI.2.13], it suffices to show that $\lim _{n \rightarrow \infty}\left|m_{T}\right|\left(O_{n}\right)=0$ uniformly in $T \in \mathcal{M}$, for all disjoint sequences $\left(O_{n}\right)$ of open subsets of $\Omega$. By contradiction, suppose that there exists $\varepsilon>0$, a sequence $\left(T_{n}\right)$ in $M$, and a strictly increasing sequence $\left(k_{n}\right)$ of natural numbers such that

$$
\left|m_{T_{n}}\right|\left(O_{k_{n}}\right)>2 \varepsilon, \quad \forall n \in \mathbb{N} .
$$


Now we consider the operators $S_{n}: \mathscr{C}\left(\Omega, O_{k_{n}}\right) \rightarrow Y$ defined by

$$
S_{n} \varphi=\int_{O_{k_{n}}} \varphi d m_{T_{n}}
$$

for all $\varphi \in \mathscr{C}\left(\Omega, O_{k_{n}}\right)$, where $\mathscr{C}\left(\Omega, O_{k_{n}}\right)$ is the closed subspace of $\mathscr{C}(\Omega)$ formed by all continuous functions $\varphi$ on $\Omega$ such that $\varphi$ vanishes in $\Omega \backslash O_{k_{n}}$. It is known that $\pi_{1}\left(S_{n}\right)=$ $\left|m_{T_{n}}\right|\left(O_{k_{n}}\right)$, for all $n \in \mathbb{N}$ [7, Theorem 19.3]. For each $n \in \mathbb{N}$, we can choose a finite set $\left\{\varphi_{1}^{n}, \ldots, \varphi_{p_{n}}^{n}\right\} \subset \mathscr{C}\left(\Omega, O_{k_{n}}\right)$ satisfying $\epsilon_{1}\left(\varphi_{i}^{n}\right)_{i=1}^{p_{n}} \leq 1$ and

$$
\sum_{i=1}^{p_{n}}\left\|S_{n} \varphi_{i}^{n}\right\|>\pi_{1}\left(S_{n}\right)-\varepsilon
$$

Since the open sets $O_{k_{n}}$ are disjoint, it follows that the sequence $\left(\varphi_{1}^{1}, \ldots, \varphi_{p_{1}}^{1}, \varphi_{1}^{2}, \ldots\right.$, $\left.\varphi_{p_{2}}^{2}, \ldots\right)$ belongs to $\ell_{w}^{1}(\mathscr{C}(\Omega))$. Nevertheless, for all $n \in \mathbb{N}$, we have

$$
\sum_{m \geq n} \sum_{i=1}^{p_{m}}\left\|T_{n} \varphi_{i}^{m}\right\| \geq \sum_{i=1}^{p_{n}}\left\|T_{n} \varphi_{i}^{n}\right\|=\sum_{i=1}^{p_{n}}\left\|S_{n} \varphi_{i}^{n}\right\|>\pi_{1}\left(S_{n}\right)-\varepsilon=\left|m_{T_{n}}\right|\left(O_{k_{n}}\right)-\varepsilon>\varepsilon .
$$

This denies (a) and proves that (a) implies (b).

(b) $\Rightarrow$ (c). Again we proceed by contradiction. Suppose $\left(E_{n}\right)$ is a disjoint sequence of Borel subsets of $\Omega$ for which there exists $\varepsilon>0$, a sequence $\left(T_{n}\right)$ in $\mathcal{M}$, and a strictly increasing sequence $\left(k_{n}\right)$ of natural numbers so that

$$
\sum_{i=k_{n}+1}^{k_{n+1}}\left\|m_{T_{n}}\left(E_{i}\right)\right\|>\varepsilon, \quad \forall n \in \mathbb{N}
$$

If we put $B_{n}=\bigsqcup_{i=k_{n}+1}^{k_{n+1}} E_{i}$, the above inequality yields $\left|m_{T_{n}}\right|\left(B_{n}\right)>\varepsilon$. So, in view of [6, Proposition I.1.17], the family $\left\{\left|m_{T}\right|: T \in \mathcal{M}\right\}$ is not uniformly countably additive.

(c) $\Rightarrow(b)$. We need to prove

$$
\lim _{n \rightarrow \infty}\left|m_{T}\right|\left(E_{n}\right)=0 \quad \text { uniformly in } T \in \mathcal{M},
$$

for all disjoint sequences $\left(E_{n}\right)$ of Borel subsets of $\Omega$. Suppose (b) fails. Then, there exists $\varepsilon>0$, a sequence $\left(T_{n}\right)$ in $\mathcal{M}$, and a strictly increasing sequence $\left(k_{n}\right)$ of natural numbers satisfying

$$
\left|m_{T_{n}}\right|\left(E_{k_{n}}\right)>\varepsilon, \quad \forall n \in \mathbb{N} .
$$

For each $n \in \mathbb{N}$, we choose a finite partition $\left\{E_{1}^{n}, \ldots, E_{p_{n}}^{n}\right\}$ of $E_{k_{n}}$ for which

$$
\sum_{i=1}^{p_{n}}\left\|m_{T_{n}}\left(E_{i}^{n}\right)\right\|>\varepsilon
$$

Then, the disjoint sequence $\left(E_{1}^{1}, \ldots, E_{p_{1}}^{1}, E_{1}^{2}, \ldots, E_{p_{2}}^{2}, \ldots\right)$ does not satisfy (c). 
(b) $\Rightarrow$ (a). According to [6, Theorem I.2.4] there exists a countably additive measure $\mu: \Sigma \rightarrow[0, \infty)$ so that

$$
\lim _{\mu(E) \rightarrow 0}\left|m_{T}\right|(E)=0 \quad \text { uniformly in } T \in \mathcal{M} .
$$

Hence, given $\varepsilon>0$, there exists $\delta>0$ such that, if $E \in \Sigma$ verifies $\mu(E)<\delta$, then $\left|m_{T}\right|(E)<\varepsilon / 2$, for all $T \in \mathcal{M}$.

Next, given $\left(\varphi_{n}\right) \in \ell_{w}^{1}(\mathscr{C}(\Omega))$ with $\epsilon_{1}\left(\varphi_{n}\right) \leq 1$, notice that the series $\sum_{n=1}^{\infty}\left|\varphi_{n}(t)\right|$ is convergent for all $t \in \Omega$. Put $f_{n}(t)=\sum_{k=1}^{n}\left|\varphi_{k}(t)\right|$ and $f(t)=\lim _{n \rightarrow \infty} f_{n}(t)$. By Egorov's theorem, the sequence $\left(f_{n}\right)$ is quasi-uniformly convergent to $f$. Then, there exists $E \in \Sigma$ such that $\mu(E)<\delta$ and

$$
f_{n \mid \Omega \backslash E} \longrightarrow f_{\mid \Omega \backslash E}
$$

uniformly. If $C=\sup \left\{\left|m_{T}\right|(\Omega): T \in \mathcal{M}\right\}$, there exists $n_{0} \in \mathbb{N}$ so that

$$
\sum_{n \geq n_{0}}\left|\varphi_{n}(t)\right|<\frac{\varepsilon}{2 C}, \quad \forall t \in \Omega \backslash E
$$

Now,

$$
\begin{aligned}
\sum_{n \geq n_{0}}\left\|T \varphi_{n}\right\| & =\sum_{n \geq n_{0}}\left\|\int_{\Omega} \varphi_{n}(t) d m_{T}\right\| \\
& \leq \sum_{n \geq n_{0}}\left\|\int_{E} \varphi_{n}(t) d m_{T}\right\|+\sum_{n \geq n_{0}}\left\|\int_{\Omega \backslash E} \varphi_{n}(t) d m_{T}\right\| \\
& \leq \sum_{n \geq n_{0}} \int_{E}\left|\varphi_{n}(t)\right| d\left|m_{T}\right|+\sum_{n \geq n_{0}} \int_{\Omega \backslash E}\left|\varphi_{n}(t)\right| d\left|m_{T}\right| \\
& =\int_{E}\left(\sum_{n \geq n_{0}}\left|\varphi_{n}(t)\right|\right) d\left|m_{T}\right|+\int_{\Omega \backslash E}\left(\sum_{n \geq n_{0}}\left|\varphi_{n}(t)\right|\right) d\left|m_{T}\right| \\
& \leq\left|m_{T}\right|(E)+\frac{\varepsilon}{2 C}\left|m_{T}\right|(\Omega \backslash E) \\
& <\varepsilon .
\end{aligned}
$$

We denote by $\mathscr{V}(X, Y)$ the class of completely continuous operators from $X$ into $Y$, that is, the class of operators which map weakly convergent sequences in $X$ into norm-convergent sequences in $Y$. A set $\mu \subset \mathscr{V}(X, Y)$ is said to be uniformly completely continuous if, given a weakly convergent sequence $\left(x_{n}\right)$ in $X,\left(T x_{n}\right)$ is norm convergent uniformly in $T \in \mathcal{M}$. The following result gives some characterizations of uniformly completely continuous sets in $\mathscr{V}(\mathscr{C}(\Omega), Y)$. Recall that an operator $T$ defined on $\mathscr{C}(\Omega)$ is completely continuous if and only if $T$ is weakly compact [6, Corollary VI.2.17], so $m_{T}$ is countably additive and regular, too.

THEOREM 2.5. Let $\mathcal{M} \subset \mathscr{V}(\mathscr{C}(\Omega), Y)$ be a bounded set for the operator norm. The following statements are equivalent:

(a) $\mathcal{M}$ is uniformly completely continuous,

(b) the family $\left\{m_{T}: T \in \mathcal{M}\right\}$ is uniformly countably additive, 
(c) $\mathcal{M}^{*}=\left\{T^{*}: T \in \mathcal{M}\right\}$ is collectively weakly compact, that is, the set $\bigcup_{T \in \mathcal{M}} T^{*}\left(B_{Y^{*}}\right)$ is relatively weakly compact in $\mathscr{b}(\Omega)^{*}$.

Proof. (a) $\Rightarrow\left(\right.$ b). By [6, Proposition I.1.17], the family $\left\{m_{T}: T \in M\right\}$ is uniformly countably additive if and only if $\mathcal{N}=\left\{y^{*} \circ m_{T}: T \in \mathcal{M}, y^{*} \in B_{Y^{*}}\right\}$ is. According to [6, Lemma VI.1.13], we have to prove that

$$
\lim _{n \rightarrow \infty} y^{*} \circ m_{T}\left(O_{n}\right)=0 \quad \text { uniformly in } \mathcal{N},
$$

for all disjoint sequences $\left(O_{n}\right)$ of open subsets of $\Omega$. By contradiction, suppose there exists such a sequence $\left(O_{n}\right)$ for which $\lim _{n \rightarrow \infty} y^{*} \circ m_{T}\left(O_{n}\right)=0$ but not uniformly in $\mathcal{N}$. Then, there exists $\varepsilon>0$ and sequences $\left(y_{n}^{*}\right) \subset B_{Y^{*}},\left(T_{n}\right) \in \mathcal{M}$, and $\left(O_{k_{n}}\right) \subset\left(O_{n}\right)$ such that

$$
\left|y_{n}^{*} \circ m_{T_{n}}\left(O_{k_{n}}\right)\right|>\varepsilon, \quad \forall n \in \mathbb{N} .
$$

Now, using the regularity of each $m_{T_{n}}$, we can find a sequence of compact sets $\left(H_{n}\right)$ with $H_{n} \subset O_{k_{n}}$ and

$$
\left\|m_{T_{n}}\right\|\left(O_{k_{n}} \backslash H_{n}\right)<\frac{\varepsilon}{2}, \quad \forall n \in \mathbb{N}
$$

( $\|m\|$ denotes the semivariation of $m$, that is, $\|m\|(E)=\sup \left\{\left|y^{*} \circ m\right|(E): y^{*} \in B_{Y^{*}}\right\}$ ). By Urysohn's lemma, for every $n \in \mathbb{N}$ there exists a continuous function $\varphi_{n}: \Omega \rightarrow$ $[0,1]$ such that $\varphi_{n}\left(H_{n}\right)=1$ and $\varphi_{n}\left(\Omega \backslash O_{k_{n}}\right)=0$. Obviously, the series $\sum_{n=1}^{\infty} \varphi_{n}$ is unconditionally convergent in $\mathscr{C}(\Omega)$. Since $\mathcal{M}$ is uniformly completely continuous, there exists $n_{0} \in \mathbb{N}$ such that

$$
\left\|T \varphi_{n}\right\|<\frac{\varepsilon}{2}, \quad \forall n \geq n_{0}, \forall T \in \mathcal{M}
$$

Then, we have

$$
\begin{aligned}
\left\|m_{T_{n}}\left(O_{k_{n}}\right)\right\| & \leq\left\|m_{T_{n}}\left(O_{k_{n}}\right)-T_{n} \varphi_{n}\right\|+\left\|T_{n} \varphi_{n}\right\| \\
& =\left\|\int_{\Omega} x O_{k_{n}} d m_{T_{n}}-\int_{\Omega} \varphi_{n} d m_{T_{n}}\right\|+\left\|T_{n} \varphi_{n}\right\| \\
& =\left\|\int_{O_{k_{n}}}\left(1-\varphi_{n}\right) d m_{T_{n}}\right\|+\left\|T_{n} \varphi_{n}\right\| \\
& =\left\|\int_{O_{k_{n}} \backslash H_{n}}\left(1-\varphi_{n}\right) d m_{T_{n}}\right\|+\left\|T_{n} \varphi_{n}\right\| \\
& \leq\left\|m_{T_{n}}\right\|\left(O_{k_{n}} \backslash H_{n}\right)+\left\|T_{n} \varphi_{n}\right\| \\
& <\varepsilon,
\end{aligned}
$$

for all $n \geq n_{0}$. This is in contradiction with (2.16).

(b) $\Rightarrow$ (a). By [6, Theorem I.2.4], there exists a scalar countably additive measure $\mu: \Sigma \rightarrow$ $[0, \infty)$ such that $\left\{m_{T}: T \in \mathcal{M}\right\}$ is uniformly $\mu$-continuous. Then, if $\left(\varphi_{n}\right)$ is a sequence 
that tends to zero weakly in $\mathscr{C}(\Omega)$, it is obvious that zero is the pointwise limit of the sequence $\left(\varphi_{n}(t)\right)$. Now, using Egorov's theorem and proceeding along similar lines as the proof of $(b) \Rightarrow(a)$ in Theorem 2.4, the proof concludes.

(b) $\Leftrightarrow$ (c). The set $\bigcup_{T \in \mathcal{M}} T^{*}\left(B_{Y^{*}}\right)=\left\{y^{*} \circ m_{T}: T \in \mathcal{M}, y^{*} \in B_{Y^{*}}\right\} \subset \mathscr{C}(\Omega)^{*}$ is relatively weakly compact if and only if it is bounded and uniformly countably additive [4, Theorem VII.13]. A call to [6, Proposition I.1.17] makes clear that $\bigcup_{T \in \mathcal{M}} T^{*}\left(B_{Y^{*}}\right)$ is uniformly countably additive if and only if condition (b) is satisfied.

COROLlary 2.6. If $\mu \subset \Pi_{1}(\mathscr{C}(\Omega), Y)$ is uniformly 1-summing, then $\mu$ is uniformly completely continuous.

The converse of the last result is not true in general.

Proposition 2.7. Suppose that the cardinal of $\Omega$ is infinite. The following statements are equivalent:

(a) each subset of $\Pi_{1}(\mathscr{b}(\Omega), Y)$ uniformly completely continuous is uniformly 1 -summing,

(b) $Y$ is finite-dimensional.

Proof. (a) $\Rightarrow($ b). By contradiction, suppose there is an unconditionally summable serie $\sum_{k} y_{k}$ in $Y$ such that $\sum_{k}\left\|y_{k}\right\|=\infty$. Let $\left(\omega_{k}\right)$ be a sequence in $\Omega$ with $\omega_{k} \neq \omega_{l}$ when $k \neq l$. For each $m \in \mathbb{N}$ consider the operator $T_{m}: \mathscr{C}(\Omega) \rightarrow Y$ defined by

$$
T_{m} \varphi=\sum_{k=1}^{m} \varphi\left(\omega_{k}\right) y_{k} .
$$

It is not difficult to show that $\mathcal{M}=\left(T_{m}\right)$ is uniformly completely continuous. Nevertheless,

$$
\pi_{1}\left(T_{m}\right)=\sum_{k=1}^{m}\left\|y_{k}\right\| \stackrel{m}{\longrightarrow} \infty,
$$

so $M$ cannot be uniformly 1 -summing because it is not $\pi_{1}$-bounded.

(b) $\Rightarrow(a)$. This follows easily in view of conditions (b) in Theorems 2.4 and 2.5.

We have showed that the converse of Corollary 2.6 is not true in general. However, a direct argument using Theorems 2.4 and 2.5 leads up to conclude that every uniformly completely continuous set $\mathcal{M} \subset \Pi_{1}(\mathscr{C}(\Omega), Y)$ verifying the following condition is uniformly 1-summing:

(i) given $T \in \mathcal{M}$ and a finite subset $\left\{\left(\varphi_{1}, y_{1}^{*}\right), \ldots,\left(\varphi_{m}, y_{m}^{*}\right)\right\}$ of $\mathscr{C}(\Omega) \times B_{Y^{*}}$, there exist $S \in \mathcal{M}$ and $z^{*} \in B_{Y^{*}}$ such that $\left|\left\langle y_{n}^{*}, T \varphi_{n}\right\rangle\right| \leq\left|\left\langle z^{*}, S \varphi_{n}\right\rangle\right|, n=1, \ldots, m$.

3. Final notes and examples. The Grothendieck-Pietsch domination theorem states that an operator $T: X \rightarrow Y$ is $p$-summing if and only if there exists a positive Radon measure $\mu$ defined on the (weak*) compact space $B_{X^{*}}$ such that

$$
\|T x\|^{p} \leq \int_{B_{X^{*}}}\left|\left\langle x^{*}, x\right\rangle\right|^{p} d \mu\left(x^{*}\right),
$$


for all $x \in X$ [5, Theorem 2.12]. Since the appearance of this theorem, there is a great interest in finding out the structure of uniformly $p$-dominated sets. A subset $\mu$ of $\Pi_{p}(X, Y)$ is uniformly $p$-dominated if there exists a positive Radon measure $\mu$ such that the inequality (3.1) holds for all $x \in X$ and all $T \in \mathcal{M}$. In [3, 8, 9], the reader can find some of the most recent steps given on this subject. Now we are going to show that these sets are uniformly $p$-summing.

Proposition 3.1. If $\mathcal{M} \subset \Pi_{p}(X, Y)$ is a uniformly $p$-dominated set, then $\mathcal{M}^{* *}=$ $\left\{T^{* *}: T \in \mathcal{M}\right\}$ is uniformly $p$-summing.

Proof. Let $\mu$ be a measure for which $\mu$ is uniformly $p$-dominated. In a similar way as in the Pietsch factorization theorem [5, Theorem 2.13], we can obtain, for all $T \in \mathcal{M}$, operators $U_{T}: L_{p}(\mu) \rightarrow \ell_{\infty}\left(B_{Y^{*}}\right),\left\|U_{T}\right\| \leq \mu\left(B_{X^{*}}\right)^{1 / p}$, and an operator $V: X \rightarrow L_{\infty}(\mu)$ such that the following diagram is commutative:

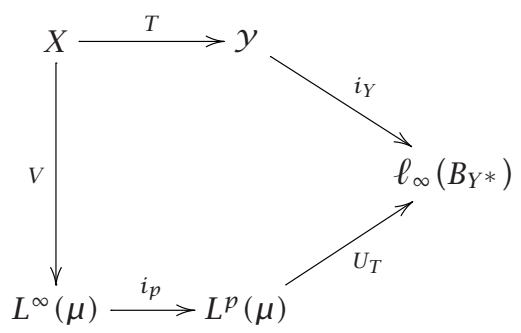

Here, $i_{p}$ is the canonical injection from $L_{\infty}(\mu)$ into $L_{p}(\mu)$ and $i_{Y}$ is the isometry from $Y$ into $\ell_{\infty}\left(B_{Y^{*}}\right)$ defined by $i_{Y}(y)=\left(\left\langle y^{*}, y\right\rangle\right)_{y^{*} \in B_{Y^{*}}}$. Notice that $i_{p}^{* *}$ can be viewed as $i_{p}$ composed with the canonical projection $P: L_{\infty}(\mu)^{* *} \rightarrow L_{\infty}(\mu)$ which is simply the adjoint of the usual embedding $L_{1}(\mu) \rightarrow L_{1}(\mu)^{* *}$. By weak compactness, we may and do consider $T^{* *}$ as a map from $X^{* *}$ into $Y$ for which

$$
i_{Y} \circ T^{* *}=U_{T} \circ i_{p} \circ P \circ V^{* *} .
$$

Given $\varepsilon>0$ and $\left(x_{n}^{* *}\right) \in \ell_{w}^{p}\left(X^{* *}\right)$, we can choose $n_{0} \in \mathbb{N}$ so that

$$
\sum_{n \geq n_{0}}\left\|i_{p} \circ P \circ V^{* *}\left(x_{n}^{* *}\right)\right\|^{p}<\frac{\varepsilon}{\mu\left(B_{X^{*}}\right)}
$$

because $i_{p} \circ P \circ V^{* *}$ is $p$-summing. Then, we have

$$
\begin{aligned}
\sum_{n \geq n_{0}}\left\|T^{* *} x_{n}^{* *}\right\|^{p} & =\sum_{n \geq n_{0}}\left\|i_{Y} \circ T^{* *}\left(x_{n}^{* *}\right)\right\|^{p}=\sum_{n \geq n_{0}}\left\|U_{T} \circ i_{p} \circ P \circ V^{* *}\left(x_{n}^{* *}\right)\right\|^{p} \\
& \leq \mu\left(B_{X^{*}}\right) \sum_{n \geq n_{0}}\left\|i_{p} \circ P \circ V^{* *}\left(x_{n}^{* *}\right)\right\|^{p}<\varepsilon,
\end{aligned}
$$

for all $T \in \mathcal{M}$. So, $\mathcal{M}^{* *}$ is uniformly $p$-summing. 
It is easy to show that the study of uniformly $p$-summing sets can be reduced to the behavior of its sequences. Indeed, a bounded set $\mu$ in $\Pi_{p}(X, Y)$ is uniformly $p$ summing if and only if every sequence $\left(T_{n}\right)$ in $\mathcal{M}$ admits a uniformly $p$-summing subsequence. Thus, it seems to be interesting to make clear the relationship between uniformly $p$-summing sets and relatively weakly compact sets. For $p=1$, we have the following result.

Proposition 3.2. Every relatively weakly compact set in $\Pi_{1}(X, Y)$ is uniformly 1summing.

Proof. Let $\mu$ be a relatively weakly compact set in $\Pi_{1}(X, Y)$. Given $\hat{x}=\left(x_{n}\right) \in$ $\ell_{w}^{1}(X)$, consider the (weak-weak) continuous operator $U_{\hat{x}}: \Pi_{1}(X, Y) \rightarrow \ell_{a}^{1}(Y)$ defined by $U_{\hat{x}}(T)=\left(T x_{n}\right)$. Then, $U_{\hat{x}}(\mathcal{M})$ is relatively weakly compact in $\ell_{a}^{1}(Y)$; according to [2, Theorem 2], we can conclude that $\mathcal{M}$ is uniformly 1 -summing.

Proposition 3.2 does not remain true if $p=2$. For example, for each $\beta=\left(\beta_{n}\right) \in \ell_{2}$ consider the operator $T_{\beta}: c_{0} \rightarrow \ell_{2}$ defined by $T\left(\alpha_{n}\right)=\left(\alpha_{n} \cdot \beta_{n}\right)$ and put $\mathcal{M}=\left\{T_{\beta}: \beta \in\right.$ $\left.B_{\ell_{2}}\right\} \subset \Pi_{2}\left(c_{0}, \ell_{2}\right)$ [5, Theorem 3.5]. If we consider $\ell_{2}$ as a subspace of $\Pi_{2}\left(c_{0}, \ell_{2}\right)$, the set $\mathcal{M}=B_{\ell_{2}}$ is relatively weakly compact. Nevertheless, no matter how we choose $k \in \mathbb{N}$,

$$
\sum_{n \geq k}\left\|T_{e_{k}} e_{n}\right\|^{2}=1
$$

so $\mathcal{M}$ cannot be uniformly 2-summing.

Now we show that there are uniformly $p$-summing sets failing to be relatively weakly compact.

Proposition 3.3. If every uniformly $p$-summing set is relatively weakly compact in $\Pi_{p}(X, Y)$, then $Y$ is reflexive.

Proof. Fixing $x_{0}^{*} \in X^{*}$ with $\left\|x_{0}^{*}\right\|=1$, the isometry $y \in Y \mapsto x_{0}^{*} \otimes y \in x_{0}^{*} \otimes Y$ allows us to see $Y$ as a subspace of $\Pi_{p}(X, Y)$. If $\varepsilon>0$ and $\left(x_{n}\right) \in \ell_{w}^{p}(X)$, there exists $n_{0} \in \mathbb{N}$ so that

$$
\sum_{n \geq n_{0}}\left|\left\langle x_{0}^{*}, x_{n}\right\rangle\right|^{p}<\varepsilon
$$

hence, for every $y \in B_{Y}$,

$$
\sum_{n \geq n_{0}}\left\|\left(x_{0}^{*} \otimes y\right)\left(x_{n}\right)\right\|^{p}=\sum_{n \geq n_{0}}\left|\left\langle x_{0}^{*}, x_{n}\right\rangle\right|^{p}\|y\|^{p}<\varepsilon .
$$

This yields that $B_{Y}$ is uniformly $p$-summing and, by hypothesis, weakly compact.

The converse of Proposition 3.3 is not always true. By contradiction, suppose every uniformly 1 -summing set in $\Pi_{1}\left(\ell_{1}, \ell_{2}\right)$ is relatively weakly compact. Because $\ell_{1}$ does not contain any copy of $c_{0}$, every bounded set in $\Pi_{1}\left(\ell_{1}, \ell_{2}\right)$ is relatively weakly compact. Then, we conclude that $\Pi_{1}\left(\ell_{1}, \ell_{2}\right)$ is reflexive, which is not possible since $\ell_{1}^{*}$, viewed as a subspace of $\Pi_{1}\left(\ell_{1}, \ell_{2}\right)$, is not.

However, if $p=1$ and $X=\mathscr{C}(\Omega)$, the reflexivity of $Y$ is a sufficient condition for a uniformly 1-summing set to be relatively weakly compact. Indeed, if $r b v c a(\Sigma, Y)$ denotes 
the set of all regular, countably additive, $Y$-valued measures $m$ on $\Sigma$ with bounded variation, recall that relatively weakly compact sets $\mathcal{M}$ in $\operatorname{rbvca}(\Sigma, Y)$ are those verifying the following conditions: (i) $\mathcal{M}$ is bounded; (ii) the family of nonnegative measures $\{|m|: m \in \mathcal{M}\}$ is uniformly countably additive; and (iii) for each $E \in \Sigma$, the set $\{m(E): m \in \mathcal{M}\}$ is relatively weakly compact in $Y$ [6, Theorem IV.2.5]. Having in mind the identification between $\Pi_{1}(\mathscr{C}(\Omega), Y)$ and $\operatorname{rbvca}(\Sigma, Y)$, and making use of the characterization of uniformly 1 -summing sets obtained in Theorem 2.4 , we conclude the next characterization.

COROLLARY 3.4. The following statements are equivalent:

(a) $Y$ is reflexive,

(b) every set $\mathcal{M}$ in $\Pi_{1}(\mathscr{C}(\Omega), Y)$ is uniformly 1 -summing if and only if $\mathcal{M}$ is relatively weakly compact.

It is well known that a linear operator $T$ is 1 -summing if and only if $T^{* *}$ is. So, it is natural to ask if a set $\mathcal{M}$ is uniformly 1 -summing whenever $\mathcal{M}^{* *}=\left\{T^{* *}: T \in \mathcal{M}\right\}$ is. Unfortunately, we are going to show that this is not true in general. It suffices to take $X$ as the separable $\mathscr{L}_{\infty}$-space of Bourgain and Delbaen [1]. This space has the RadonNikodym property, so it does not contain any copy of $c_{0}$. Nevertheless, $X^{*}$ is isomorphic to $\ell_{1}$ and, therefore, $X^{* *}$ contains a copy of $c_{0}$. Let $\left(e_{n}\right)$ be the canonical basis of $\ell_{1}$ and $J: \ell_{1} \rightarrow X^{*}$ an isomorphism. Put $T_{n}=J e_{n} \in \Pi_{1}(X, \mathbb{R})$; the set $\mathcal{M}=\left\{T_{n}: n \in \mathbb{N}\right\}$ is uniformly 1-summing since it is bounded and $X$ does not contain any copy of $c_{0}$. Notice that the elements of $\mu^{* *}$ are the linear forms $x^{* *} \in X^{* *} \mapsto\left\langle x^{* *}, J e_{n}\right\rangle \in \mathbb{R}$, for all $n \in \mathbb{N}$. If $\left(e_{n}^{*}\right)$ is the canonical basis of $c_{0}$, then $\left(\left(J^{*}\right)^{-1}\left(e_{n}^{*}\right)\right) \in \ell_{w}^{1}\left(X^{* *}\right)$; hence, no matter how we choose $k \in \mathbb{N}$, it turns out that

$$
\sum_{n \geq k}\left|T_{k}^{* *}\left(\left(J^{*}\right)^{-1}\left(e_{n}^{*}\right)\right)\right|=\sum_{n \geq k}\left|\left\langle\left(J^{*}\right)^{-1}\left(e_{n}^{*}\right), J e_{k}\right\rangle\right|=\sum_{n \geq k}\left|\left\langle e_{n}^{*}, e_{k}\right\rangle\right|=1,
$$

and $\mu^{* *}$ cannot be uniformly 1 -summing.

Nevertheless, if $\mathcal{M}$ is a set of operators defined on $c_{0}$, then it is true that $\mathcal{M}$ is uniformly 1 -summing if and only if $\mu^{* *}$ is too. To see this, notice that for a 1-summing operator $T$ : $\left(\alpha_{n}\right) \in c_{0} \mapsto \sum_{n=1}^{\infty} \alpha_{n} x_{n} \in X$, the second adjoint $T^{* *}: \ell_{\infty} \rightarrow X$ is defined by $T^{* *}\left(\beta_{n}\right)=$ $\sum_{n=1}^{\infty} \beta_{n} x_{n}$, for all $\left(\beta_{n}\right) \in \ell_{\infty}$.

When $\mathcal{M}$ is a set of operators defined on a $\mathscr{C}(\Omega)$-space, we do not know whether $\mathcal{M}^{* *}$ inherits the property or not. Anyway, we are going to prove the following weaker result. We inject isometrically $B(\Sigma)$ into $\mathscr{C}(\Omega)^{* *}$ in the natural way.

Proposition 3.5. If $\mu \subset \Pi_{1}(\mathscr{C}(\Omega), X)$ is uniformly 1-summing, then $\tilde{\mathcal{M}}=\{\tilde{T}: B(\Sigma) \rightarrow$ $X: T \in \mathcal{M}\}$ is uniformly 1 -summing too.

Proof. The argument is similar to the one used in the proof of $(b) \Rightarrow(a)$ in Theorem 2.4.

Finally, we give an example to show that Corollary 2.6 is not true if $\mathscr{C}(\Omega)$ is replaced by a general Banach space $X$. It suffices to take $X=\ell_{2}$ and $\mathcal{M}=\left\{e_{n}^{*}: n \in \mathbb{N}\right\}$, where $\left(e_{n}^{*}\right)$ is the unit basis of $\ell_{2}^{*} \simeq \ell_{2}$. The set $\mathcal{M}$ is bounded in $\Pi_{1}\left(\ell_{2}, \mathbb{R}\right)$ and, therefore, uniformly 1 -summing but it is not uniformly completely continuous. 


\section{REFERENCES}

[1] J. Bourgain and F. Delbaen, A class of special $\mathscr{L}_{\infty}$-spaces, Acta Math. 145 (1980), no. 3-4, 155-176.

[2] J. K. Brooks and N. Dinculeanu, Weak compactness in spaces of Bochner integrable functions and applications, Adv. Math. 24 (1977), no. 2, 172-188.

[3] J. M. Delgado and C. Piñeiro, A note on uniformly dominated sets of summing operators, Int. J. Math. Math. Sci. 29 (2002), no. 5, 307-312.

[4] J. Diestel, Sequences and Series in Banach Spaces, Graduate Texts in Mathematics, vol. 92, Springer-Verlag, New York, 1984.

[5] J. Diestel, H. Jarchow, and A. Tonge, Absolutely Summing Operators, Cambridge Studies in Advanced Mathematics, vol. 43, Cambridge University Press, Cambridge, 1995.

[6] J. Diestel and J. J. Uhl, Jr., Vector Measures, Mathematical Surveys, vol. 15, American Mathematical Society, Rhode Island, 1977.

[7] N. Dinculeanu, Vector Measures, International Series of Monographs in Pure and Applied Mathematics, vol. 95, Pergamon Press, Oxford; VEB Deutscher Verlag der Wissenschaften, Berlin, 1967.

[8] R. Khalil and M. Hussain, Uniformly dominated sets of p-summing operators, Far East J. Math. Sci., Special Volume (1998), no. Part I, 59-68.

[9] B. Marchena and C. Piñeiro, Bounded sets in the range of an $X^{* *}$-valued measure with bounded variation, Int. J. Math. Math. Sci. 23 (2000), no. 1, 21-30.

J. M. Delgado: Departamento de Matemáticas, Facultad de Ciencias Experimentales, Campus Universitario del Carmen, Avda. de las Fuerzas Armadas, 21071 Huelva, Spain

E-mail address: jmde1ga@uhu.es

Cándido Piñeiro: Departamento de Matemáticas, Facultad de Ciencias Experimentales, Campus Universitario del Carmen, Avda. de las Fuerzas Armadas, 21071 Huelva, Spain

E-mail address: candi do@uhu.es 


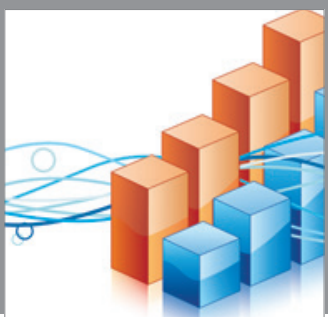

Advances in

Operations Research

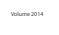

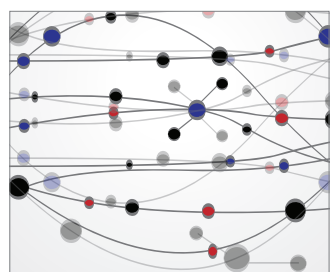

\section{The Scientific} World Journal
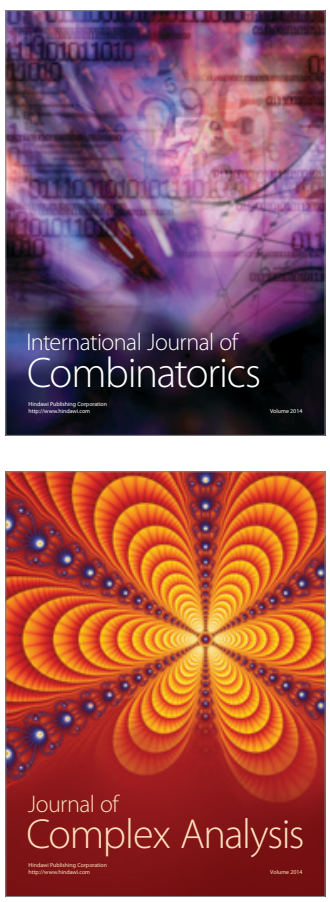

International Journal of

Mathematics and

Mathematical

Sciences
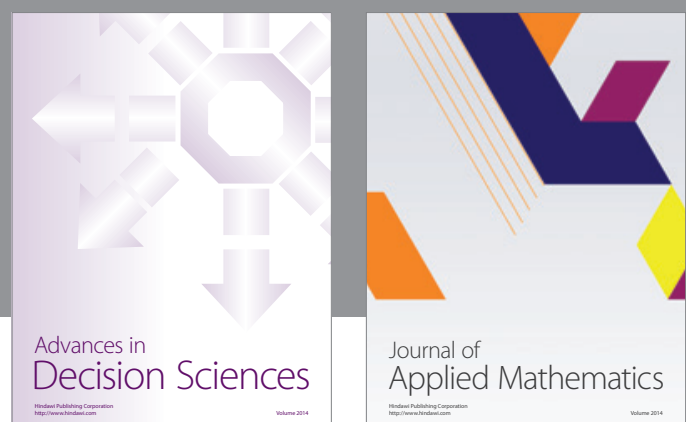

Journal of

Applied Mathematics
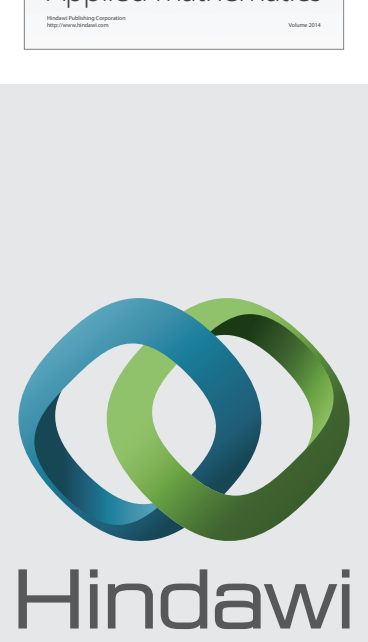

Submit your manuscripts at http://www.hindawi.com
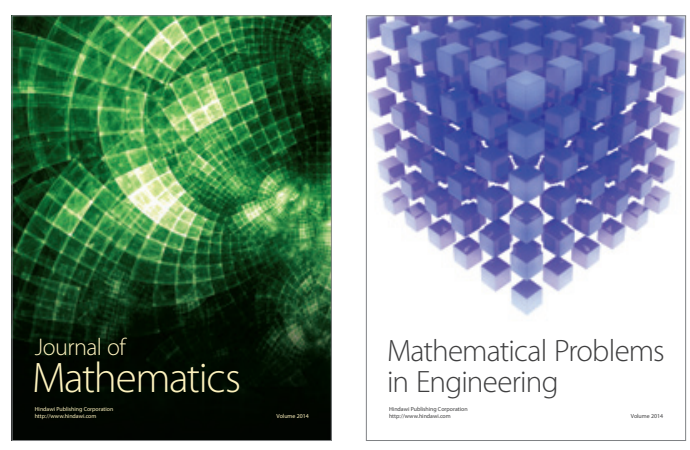

Mathematical Problems in Engineering
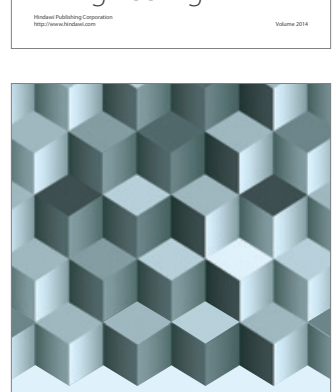

Journal of

Function Spaces
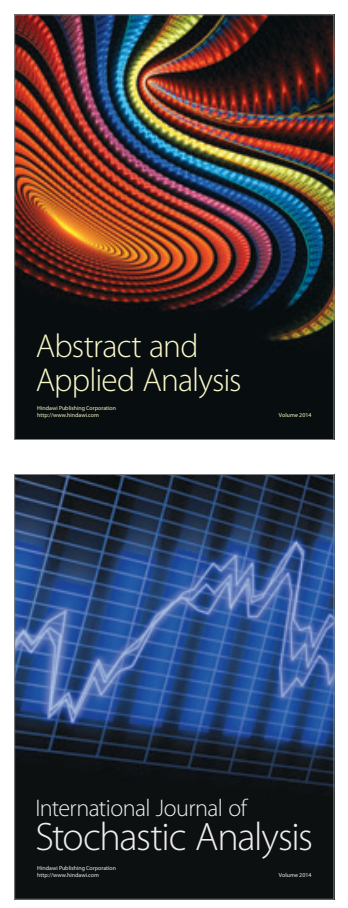

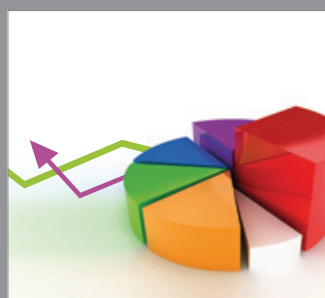

ournal of

Probability and Statistics

Promensencen
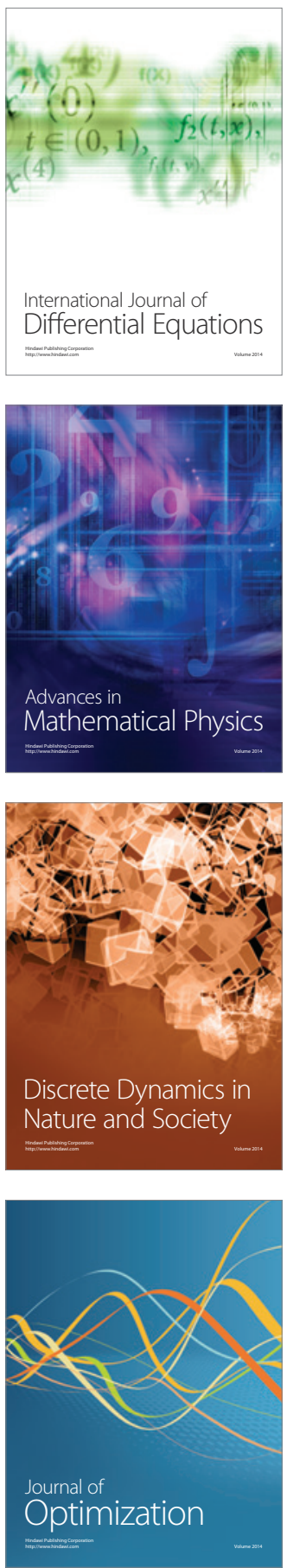